\title{
Alterações no funcionamento do sistema de controle postural de idosos. Uso da informação visual
}

\author{
Paulo Freitas Júnior \\ José A. Barela \\ https://doi.org/10.5628/rpcd.06.01.94
}

\author{
Universidade Estadual Paulista \\ Departamento de Educação Física \\ Instituto de Biociências \\ Laboratório para Estudos do Movimento \\ Rio Claro \\ Brasil
}

\section{RESUMO}

Com o avanço da idade, os seres humanos apresentam alterações no controle postural. Tais alterações têm sido consideradas ocorrer em função da diminuição da capacidade dos sistemas sensoriais em fornecer informações e do sistema motor em produzir ações motoras adequadas para manter o corpo equilibrado e em uma posição desejada. Estas alterações levariam a uma diminuição no desempenho do sistema de controle postural e estariam associadas ao aumento da incidência de quedas em idosos. Todavia, essa relação entre alterações sensoriais e motoras e a diminuição no desempenho do sistema de controle postural ainda não é bem compreendida. E, mais importante, os maiores problemas para o funcionamento do sistema de controle postural poderiam não estar associados a alterações em cada um desses sistemas, mas sim, poderiam estar relacionados a alterações no relacionamento entre informação sensorial e ação motora, que ocorrem em idosos. Assim, este trabalho de revisão visa apresentar e discutir os principais aspectos do controle postural, com ênfase no relacionamento entre informação visual e ação motora, e como estes aspectos podem auxiliar o entendimento das alterações observadas no controle postural em idosos.

Palavras-chave: controle postural, equilíbrio, envelhecimento, acoplamento percepção-ação, idosos, sala móvel.

\section{ABSTRACT}

Changes in Elderly Postural Control System Functioning. Use of Visual Information

Aging process has led human beings to exhibit changes in postural control. Such changes have been considered to occur due to problems in the sensory systems to provide information about body position on space, and also to problems in the motor system to produce adequate and coordinate muscle activation to maintain the body in a desirable position. These changes would lead elderly people to decline their performance in postural control and would be associated to the increase of incidence in falls of this population. However, this relationship between sensory and motor changes, and the decrease of the performance in postural control is poorly understood; more important, functioning problems of the postural control system would not be related to changes on each one of these systems. It could be related with changes in the relationship between sensory information and motor action in elderly people during the maintenance of the upright stance. Thus, the purposes of this paper are to present and to argue main aspects related to postural control, with emphasis in the relationship between visual information and motor action, and how these aspects can help us to understand the changes observed in the postural control in elderly people.

Key Words: postural control, equilibrium, aging, perception-action coupling, elderly, moving room. 


\section{INTRODUÇÃO}

O aumento da expectativa de vida e o conseqüente crescimento no número de pessoas que ultrapassa a barreira dos 60 anos de idade são fenômenos populacionais que vêm ocorrendo, há algum tempo, em uma grande parcela dos países desenvolvidos. No Brasil, a porcentagem de pessoas idosas, relativa ao total da população, também vem crescendo, de forma rápida, desde o início da década de 60 do século passado. Por exemplo, a proporção de indivíduos que ultrapassam os 60 anos de idade saltou de $25 \%$, no início do século XX, para cerca de $70 \%$, no final deste mesmo século [8]. Concomitantemente ao aumento do número de pessoas com mais de 60 anos, tem aumentado a preocupação com o bemestar geral, com a manutenção de um estilo de vida independente e autônomo, enfim, tem aumentado a preocupação com a manutenção e/ou busca de uma melhor qualidade de vida desta população.

Um dos maiores problemas enfrentados pelos idosos é o aumento da incidência de quedas ocorridas nesta população. A ocorrência de quedas é, nos dias atuais, um dos principais fatores de mortalidade e morbidade em idosos, principalmente em função de suas conseqüências (i.e. fraturas, imobilizações, perda de mobilidade, dependência para realização de atividades da vida diária, entre outras) [34]. Deste modo, entender quais os fatores que levam ao aumento do número de quedas em idosos tem despertado interesse de muitos pesquisadores da área de estudo do movimento humano.

Muitos estudiosos têm sugerido que o aumento do número de quedas em idosos estaria relacionado às alterações ocorridas no sistema de controle postural, principalmente nos sistemas sensoriais e motor [25, 34, 49, 54]. Embora esta relação seja coerente, não foi verificada uma relação direta entre alterações estruturais e funcionais nesses sistemas e diminuição no desempenho do sistema de controle postural e, também, entre tais alterações e o aumento no número de quedas em pessoas idosas consideradas saudáveis. A partir disso, algumas questões podem ser levantadas a respeito das características do controle postural em idosos: Qual (is) seria (m) a(s) causa(s) da diminuição no desempenho do sistema de controle postural em idosos? As alterações estruturais e funcionais que ocorrem nos canais senso- riais e no sistema motor com o avanço da idade seriam as principais responsáveis pela diminuição no desempenho do sistema de controle postural? Poderiam existir outros fatores que também colaborariam para esta diminuição?

Baseado nestas questões, o objetivo deste trabalho de revisão é apresentar e discutir os principais aspectos relacionados ao controle postural, com ênfase no relacionamento entre informação sensorial e ação motora, e como estes aspectos podem auxiliar no entendimento das alterações observadas no controle postural de pessoas idosas.

\section{CONTROLE POSTURAL}

De modo geral, o termo "postura" representa o posicionamento dos segmentos corporais, uns em relação aos outros, e da orientação destes segmentos no espaço [18]. Ainda, este termo abrange vários aspectos relacionados à biomecânica e ao controle motor, tais como: o controle da posição do centro de massa ${ }^{1}$ (CM) do corpo e sua relação com os limites da base de suporte; a estabilização do corpo durante a realização de movimentos voluntários; e a manutenção dos segmentos corporais em uma orientação específica com relação aos outros segmentos, ao ambiente, ou a ambos [25]. Assim, pode-se definir controle postural como sendo os processos pelos quais o sistema nervoso central (SNC) gera padrões de atividade muscular necessários para regular a relação entre o $\mathrm{CM}$ do corpo e a base de suporte [36].

De acordo com Horak e Macpherson [25], o controle postural possui dois objetivos comportamentais: $\mathrm{O}$ equilíbrio postural e a orientação postural. O equilíbrio postural está relacionado ao controle da relação entre forças externas (i.e. força gravitacional), que agem sobre o corpo, e forças internas (torques articulares), que são produzidas pelo corpo. Este controle se faz necessário, pois, as forças que atuam no corpo e/ou são produzidas pelo corpo agem acelerando-o e, conseqüentemente, fazendo com que este altere seu alinhamento e se afaste da posição desejada ou da posição considerada de maior estabilidade. Desta forma, o equilíbrio corporal é alcançado quando todas as forças que agem neste corpo, tanto externas como internas, estão controladas, o que permite que o corpo permaneça em uma posição desejada (equilíbrio estático) ou que se mova de 
uma maneira controlada (equilíbrio dinâmico). Já a orientação postural está relacionada ao posicionamento e ao alinhamento dos segmentos corporais uns em relação aos outros e em relação ao ambiente. Este posicionamento e alinhamento são alcançados por meio de ações coordenadas dos vários grupos musculares responsáveis pela manutenção da relação estabelecida entre os segmentos corporais e da relação do conjunto destes segmentos, ou do corpo como um todo, com o ambiente.

Nos últimos anos tem aumentado o interesse em entender como o ser humano mantém seu equilíbrio e orientação posturais. Segundo Horak e Macpherson [25], até algum tempo atrás, o controle postural era visto como resultado de respostas reflexas e hierarquicamente organizadas, desencadeadas por canais sensoriais independentes (i.e. reflexos de estiramento). Atualmente, o controle postural é visto como o resultado de um relacionamento complexo e dinâmico entre o sistema sensorial, constituído pelos sistemas visual, vestibular e somatossensorial, e o sistema motor [1, 2, 25, 47]. A partir desta visão, é importante conhecer como cada um destes sistemas auxilia a manutenção do equilíbrio e orientação posturais e como tais sistemas se relacionam.

Com relação ao sistema motor, pode-se afirmar que ele é responsável por gerar atividade muscular apropriada para a busca e/ou manutenção do equilíbrio corporal e de uma orientação corporal desejada. Mas como a atividade muscular apropriada é gerada durante a manutenção da postura ereta? Basicamente, a atividade muscular tônica dos músculos antigravitacionais associada à rigidez intersegmentar gerada pelos componentes passivos dos músculos (tecido conectivo elástico) e articulações (cápsula articular e ligamentos) auxiliam, de modo importante, a manutenção do alinhamento corporal durante a manutenção da postura ereta [25]. Entretanto, o corpo humano, por ser mantido ereto dentro de uma base de suporte relativamente pequena e por ter o CM do corpo posicionado a uma altura considerável, é um sistema eminentemente instável. Esta instabilidade é comprovada pelas oscilações corporais apresentadas pelos seres humanos e pela busca constante de uma posição de equilíbrio durante a manutenção da postura ereta. Deste modo, para que o corpo alcance ou se mantenha em uma posi- ção de equilíbrio e em uma orientação corporal desejada é necessário um controle ativo, por parte do sistema de controle postural, da intensidade e duração da atividade dos vários grupos musculares responsáveis pelo controle postural [25,37]. Este controle ativo dos músculos é realizado com base nos estímulos sensoriais captados continuamente durante a manutenção da postura ereta $[1,25]$.

Estes estímulos são captados principalmente pelos sistemas vestibular, somatossensorial e visual $[25$, $38,56]$, são enviados ao SNC e integrados no sistema de controle postural. O sistema vestibular baseia-se em estímulos provenientes do aparato vestibular, localizado na orelha interna, para fornecer informações ao sistema de controle postural sobre a orientação da cabeça em relação à atuação da força gravitacional, por meio das informações de aceleração linear e angular da cabeça. O sistema somatossensorial baseia-se em informações de diversos sensores espalhados por todo corpo (i.e. fusos musculares, órgãos tendinosos de Golgi, receptores articulares e cutâneos, etc.), para fornecer um conjunto de informações sobre: (a) a posição e a velocidade de todos os segmentos corporais, em relação aos outros segmentos e em relação ao ambiente, (b) o comprimento muscular e (c) o contato com objetos externos, incluindo o contato com a superfície de suporte $[25,38,56]$. O sistema visual utiliza-se de estímulos visuais para fornecer informações do ambiente e da direção e velocidade dos movimentos corporais em relação ao ambiente [38], além de diferenciar o que é auto-movimento, ou movimento do próprio corpo, do que é movimento de um objeto no ambiente [16]. Sua eficácia para o controle postural depende, basicamente, da eficiência deste sistema em detectar, por meio de alterações no fluxo óptico, movimentos corporais relativos a um determinado ambiente [41]. Apesar de cada um dos sistemas fornecer diferentes tipos de informação para o sistema de controle postural, a ação individual de cada um deles não é suficiente para obter informações acuradas da posição do CM do corpo no espaço. Para que o sistema de controle postural obtenha tal informação, os estímulos sensoriais, provenientes dos sistemas visual, vestibular e somatossensorial, devem ser integrados no sistema de controle postural, a fim de proporcionar uma acurada representação da posição e dos movi- 
mentos do CM e, deste modo, proporcionar um controle postural efetivo e flexível [25, 26, 40].

No processo de integração sensorial, as informações sensoriais são integradas pelo sistema de controle postural e pesos (valor de importância) são dados a cada uma destas informações, para tornar o controle postural um processo mais flexível, em função das constantes mudanças na relação do indivíduo com o ambiente [40]. Em situações normais, as informações sensoriais são redundantes, ou seja, fornecem informações que são coincidentes espacial e temporalmente ao sistema de controle postural. Tal redundância causa um "enriquecimento" da informação sobre o equilíbrio e a orientação corporal que facilita o funcionamento do sistema de controle postural. Entretanto, este enriquecimento não é resultado da soma de todas as informações sensoriais disponíveis. O sistema de controle postural, de forma dinâmica, atribui um peso ou valor de importância a cada tipo de informação sensorial que depende, basicamente, do contexto onde a tarefa postural está sendo executada [27]. Por exemplo, quando uma pessoa permanece em postura ereta em um ambiente com pouca ou nenhuma iluminação há uma diminuição do peso dado à informação visual e um aumento do peso dado às informações somatossensoriais e vestibulares para a indicação da posição e velocidade de deslocamento do CM do corpo. Similarmente, quando uma pessoa está posicionada sobre uma superfície instável ou deformável, que dificulta a utilização da informação somatossensorial oriunda do tornozelo, ocorre uma diminuição do peso dado a esta informação e aumento do peso dado às demais.

Em suma, durante o processo de integração das informações sensoriais, o sistema de controle postural deve receber as informações sensoriais disponíveis e, de forma dinâmica, selecionar as informações sensoriais mais relevantes dentro de um determinado contexto, fornecendo pesos diferentes a estas informações durante a manutenção da postura ereta, com o objetivo de gerar uma informação mais precisa do posicionamento dos segmentos corporais e do CM do corpo no espaço $[25,40]$.

Entretanto, em muitos casos onde o sistema de controle postural não consegue, de forma apropriada, captar e integrar as informações sensoriais disponíveis e gerar respostas motoras adequadas, podem ocorrer desequilíbrios e, em alguns casos, quedas. Com o avanço da idade tem sido observado um aumento na incidência de quedas e tem sido sugerido que as causas para este aumento seriam, principalmente, alterações estruturais e funcionais que ocorrem nos sistemas sensoriais e motor em função do processo de envelhecimento. Contudo, como alterações ocorrem em todos os sistemas (sensoriais e motor), é difícil definir quais destas alterações são as principais responsáveis pela perda da estabilidade postural em idosos. Deste modo, definir o que causaria a perda de estabilidade postural em idosos seria importante para estabelecer medidas preventivas e de intervenção. Assim, o próximo tópico buscou verificar se há associação entre as mudanças nos sistemas sensoriais e motor e as mudanças no controle postural em função do envelhecimento.

\section{CONTROLE POSTURAL EM IDOSOS}

Há um consenso que os idosos diminuem sua capacidade de controle postural. Alguns estudos têm apontado que os idosos apresentam alterações comportamentais durante a manutenção da postura ereta. Por exemplo, tem sido verificado freqüentemente que os idosos oscilam mais que os adultos jovens, tanto de olhos abertos, quanto de olhos fechados [6, 7, 9, 21, 24, 26, 35, 39, 50]. Em geral, os estudiosos sugerem que o aumento nas oscilações corporais em idosos seria considerado um indício de alterações no sistema de controle postural. Apesar de existirem outras evidências que indicam uma diminuição no desempenho do sistema de controle postural em idosos, os motivos desta diminuição, até o momento, não foram totalmente esclarecidos. Tem sido sugerido que as causas da diminuição no desempenho do controle postural em idosos estariam associadas às alterações estruturais e funcionais nos sistemas sensoriais e motor e a problemas na integração das informações sensoriais [26, 58]. Entretanto, não temos conhecimento de estudos que tenham verificado os efeitos diretos destas alterações no desempenho do sistema de controle postural. Deste modo, ainda não se sabe quais destas alterações estariam em maior ou em menor proporção influenciando a diminuição da capacidade de controle postural desta população. O que se pode sugerir é que as alterações estruturais e funcionais nos siste- 
mas sensoriais ocorridas em função do envelhecimento não seriam tão dramáticas, a ponto de alterar significativamente o comportamento do sistema de controle postural. Segundo Wolfson e colaboradores [57], as diferenças observadas entre idosos e adultos durante a manutenção da postura ereta, em um primeiro momento, não seriam causadas pelas alterações estruturais dos sistemas sensoriais que ocorrem em função do processo natural de envelhecimento, mas estariam associadas a alterações patológicas em um ou mais destes sistemas. Apenas em idades mais avançadas é que estas diferenças poderiam ser imputadas às alterações sensoriais causadas pelo processo de envelhecimento, em função destas alterações serem mais dramáticas em idades mais avançadas. Além das alterações sensoriais, o processo de envelhecimento também causa mudanças estruturais e funcionais no sistema neuromuscular [22, 52, 59]. Estas mudanças estruturais provocam, basicamente, uma diminuição dos níveis de força e um aumento no tempo para a produção de força máxima com o avanço da idade [21, 22, 52, 59]. Além de sofrerem uma redução no nível de força, os idosos apresentam uma redução na elasticidade do tecido conectivo muscular. A diminuição da capacidade elástica do músculo, somada às mudanças estruturais e funcionais das articulações sinoviais, pode levar os idosos a apresentar menor grau de flexibilidade e, conseqüentemente, menor amplitude de movimento articular [53]. Mas, qual a quantidade de força e amplitude de movimento articular necessárias para a manutenção da postura ereta? Gu e colaboradores [20] observaram que o valor máximo de torque articular do tornozelo gerado por idosos durante perturbações do equilíbrio foi maior que o torque gerado pelos adultos, ficando ao redor de 20 N.m em média. Ainda segundo Gu e colaboradores [20], este valor é muito inferior ao valor de torque máximo do tornozelo que os idosos podem produzir. Com estes resultados, estes autores concluíram que se os idosos apresentam dificuldades em controlar a postura, esta dificuldade não está relacionada à produção de torque articular e, conseqüentemente, à força muscular. Do mesmo modo, Maki e Mcllroy [36] também sugeriram que a magnitude de força muscular requerida para manutenção da postura ereta e para desencadear uma passada após uma perturbação ao equilí- brio é muito menor que a capacidade dos idosos em gerar força, ou seja, mesmo com a diminuição na capacidade de gerar força, os idosos conseguem gerar força muscular suficiente para manterem-se em postura ereta, e mesmo para responder a perturbações utilizando outras estratégias de controle. A partir destes resultados poder-se-ia sugerir que a capacidade de gerar força muscular não afetaria significativamente o controle postural de idosos. Com relação à amplitude de movimento articular, pode-se afirmar que a amplitude de movimento nas articulações do tornozelo, joelho e quadril necessária para a manutenção da postura ereta é mínima. Para se ter uma idéia, durante a manutenção da postura ereta, os deslocamentos angulares do tornozelo, joelho e quadril não ultrapassam 4 graus [17]. Deste modo, a redução na amplitude de movimento nas articulações do tornozelo, joelho e quadril também não seria um fator determinante para o controle postural em idosos.

Além das alterações sensoriais e motoras verificadas com o avanço da idade, alterações no sistema nervoso, tais como: a diminuição na velocidade de transmissão do impulso nervoso nos neurônios sensoriais e motores [12, 44]; a perda significativa de neurônios, de dendritos e redução no número de ramificações nervosas que prejudicam a comunicação entre as células nervosas; a diminuição do metabolismo cerebral; a redução da perfusão cerebral e alteração no metabolismo dos neurotransmissores [36], poderiam estar interferindo no desempenho do sistema de controle postural. A diminuição na velocidade de transmissão do impulso nervoso pelas vias aferentes e eferentes pode afetar consideravelmente o sistema de controle postural, principalmente em situações de perturbação, onde o tempo necessário para desencadear uma resposta postural é imprescindível para o sucesso da recuperação do equilíbrio.

Como se já não fosse suficiente a diminuição na velocidade de transmissão do impulso nervoso pelas vias aferentes e eferentes, os idosos enfrentam mais um problema para o controle efetivo da postura, principalmente após perturbações. Este problema seria o aumento no tempo necessário para o processamento da informação e para o início da resposta motora. Comportamentalmente, segundo Spirduso [48], os idosos apresentam um maior tempo de rea- 
ção (TR) comparado ao dos adultos e este tempo de reação tem um aumento muito maior quando a tarefa envolve um maior processamento de informação (TR de escolha e TR discriminativo). A partir disto, podemos questionar se tais alterações não estariam influenciando o modo com o qual o sistema de controle postural utilizaria as informações sensoriais para produzir atividade postural apropriada. Assim, será que a alteração no relacionamento entre informação sensorial e ação motora seria a principal causa de mudança na performance do controle postural de idosos? Para tentar responder a esta questão, devemos entender, primeiramente, como ocorre este relacionamento.

\section{RELACIONAMENTO ENTRE INFORMAÇÃO SENSORIAL E AÇÃO MOTORA PARA O CONTROLE POSTURAL}

Como foi anteriormente salientado, atualmente, o controle postural é visto como sendo resultado de uma relação complexa e dinâmica entre as informações sensoriais e as ações musculares. Entretanto, pouco se sabe sobre como este relacionamento acontece, pois, grande parte dos estudos, preocupados em entender os processos e mecanismos envolvidos no controle postural, assumem a captação do estímulo e a integração das informações sensoriais (percepção), e as ações musculares (ação) como duas entidades separadas ou não diretamente relacionadas. Na atual visão de sistema de controle postural [e.g., 1, 25] a principal premissa é que não se pode estudar a ação sem considerar a percepção e viceversa. Ambas estão intimamente ligadas e, deste modo, são indissociáveis. [e.g., 19].

Assim, acima de tudo, o desempenho do sistema de controle postural depende de um relacionamento coerente e estável entre informação sensorial e ação motora. Este relacionamento pode ser interpretado como resultado de uma dependência mútua entre o que é percebido e a ação motora executada [45], ou seja, a informação sensorial influencia as ações motoras executadas e, simultaneamente, a execução destas ações motoras altera o fluxo de informação sensorial disponível. Por exemplo, durante a manutenção da postura ereta, quando uma oscilação para a frente é detectada pelos sistemas sensoriais, essa informação é utilizada para que os músculos posteriores dos membros inferiores e tronco sejam ativa- dos e esta oscilação seja revertida. Juntamente com a ativação dos músculos posteriores, outras informações sensoriais são disponíveis. Tais informações estariam indicando uma diminuição da velocidade das oscilações corporais para a frente até ao momento da reversão da direção da oscilação. Após a reversão da direção da oscilação, o corpo é trazido para trás, pela ação dos músculos posteriores, até ao momento em que os sistemas sensoriais detectam um excesso de oscilação corporal para trás. Quando isto ocorre, os músculos anteriores são ativados e o mesmo processo é repetido. Portanto, nas situações em que esta dependência mútua entre informação sensorial e ação motora é repetida ou manifestada de forma regular, o padrão ou ciclo percepção-ação é formado [2, 45].

Este padrão ou ciclo percepção-ação emerge do acoplamento entre o que é percebido (informação sensorial) e a ação motora executada. O sistema de controle postural busca manter a estabilidade e a coerência deste relacionamento, para que este sistema esteja apto a enfrentar os vários tipos de perturbação ao qual este é continuamente exposto [46]. A coerência e a estabilidade deste relacionamento são construídas e refinadas por meio da experiência e da prática de determinada posição corporal [5]. Um dos modos de verificar este relacionamento é fornecer um estímulo sensorial contínuo e verificar o relacionamento temporal e espacial entre o estímulo sensorial e a ação motora. Isto pode ser investigado utilizando tanto o paradigma da sala móvel, que manipula a informação visual [p. ex. 4 , $10,11,42]$, quanto o paradigma da barra de toque ou do toque leve, que manipula a informação somatossensorial [e.g. 3, 28, 29].

No paradigma experimental da sala móvel, o participante permanece em postura ereta dentro de uma sala (cenário visual real ou virtual) que é movimentada de forma independente ao piso no qual o participante está posicionado. Esta movimentação do cenário visual causa alterações no fluxo óptico dos participantes posicionados dentro deste ambiente. Como conseqüência da alteração do fluxo óptico, os participantes apresentam ajustes posturais relacionados ao movimento do estímulo visual [31, 33]. A figura 1 apresenta uma representação gráfica da situação experimental denominada de Sala Móvel. 


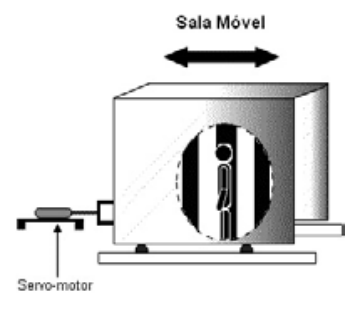

Figura 1: Representação esquemática de uma sala móvel real com destaques para o posicionamento de um participante na posição em pé, para as direções de movimento da sala e para o servo-motor que gera os movimentos.

A explicação para o efeito da manipulação visual e o desencadeamento de conseqüente oscilação corporal correspondente é que quando os participantes são submetidos aos movimentos de um cenário visual, eles procuram minimizar as alterações da imagem desse cenário projetado na retina, pois, é desse modo que o sistema de controle postural procura manter a relação entre a informação visual e o posicionamento corporal durante a manutenção da postura ereta em ambiente estacionário. Assim, quando o cenário visual é movimentado, os indivíduos produzem oscilações corporais correspondentes ao movimento do cenário visual para manter o quadro de referência estabelecido, o qual implica na manutenção da estabilidade do cenário visual projetado na retina.

Os primeiros estudos utilizando o paradigma experimental da sala móvel buscaram verificar o papel desempenhado pelo sistema visual no controle postural frente ao estímulo visual apresentado. O objetivo principal destes estudos era verificar se o estímulo visual fornecido pelo deslocamento da sala móvel desencadearia oscilações corporais em resposta a este estímulo. Em todas as faixas etárias, desde bebês recém-nascidos [30], passando por crianças $[32,55]$ e adultos $[33,34]$, até em pessoas idosas $[49,54]$, os estímulos visuais desencadearam respostas posturais coerentes e na mesma direção do estímulo. Também, por meio de movimentos contínuos de uma sala móvel, o relacionamento espacial e temporal entre informação sensorial e ação motora específica ao controle postural pôde ser verificado. A figura 2 apresenta séries temporais dos deslocamentos da sala móvel nas freqüências de 0,2, 0,5 e 0,8 $\mathrm{Hz}$ e os correspondentes deslocamentos do centro de pressão $^{2}(\mathrm{CP})$ de um adulto jovem durante a situação experimental da sala móvel.
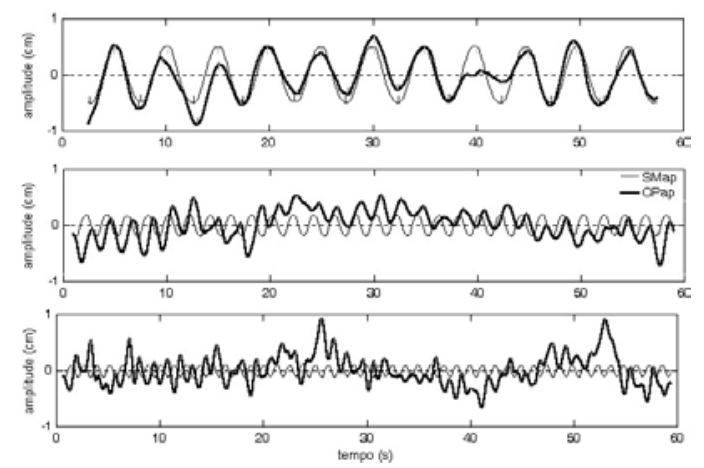

Figura 2: Deslocamento da sala móvel (linha fina) e do CP (linha grossa) na direção antero-posterior nas freqüências de 0,2 (painel superior), 0,5 (painel central) e 0,8 (painel inferior) $\mathrm{Hz}$.

Estudos para verificar como informação sensorial e ação motora estão relacionadas, utilizando para isso o paradigma da sala móvel, começaram a ser realizados a partir do final da década de 80 e início da década de 90 , do século passado $[10,11,51]$. Dijkstra e colaboradores [10] foram dos primeiros a analisar como ocorre e quais os fatores que interferem no acoplamento entre informação sensorial e ação motora. Utilizando uma sala móvel "virtual", que simulava movimentos do ambiente de forma tridimensional, estes autores verificaram que o sistema de controle postural acopla de forma coerente e estável as oscilações corporais ao estímulo visual e que o sistema de controle postural, de forma adaptativa, altera dinamicamente seus parâmetros em função das mudanças nas características do estímulo visual, buscando manter este acoplamento coerente e estável.

O segundo passo para entender como ocorre o relacionamento entre informação sensorial e ação motora veio com o estudo de Dijkstra e colaboradores [11]. Estes autores verificaram que, quando os indivíduos foram submetidos a alterações na freqüência do estímulo, o sistema de controle postural conseguiu igualar a freqüência natural de oscilação deste sistema à freqüência do estímulo visual e, ainda, conseguiu manter um acoplamento coerente e um relacionamento temporal estável entre informação visual e as oscilações corporais. Estes resultados também indicaram a ocorrência de adaptação do sistema de controle postural às características da informação visual, já que a oscilação corporal gerada pela 
estimulação óptica levou a ajustes na parte não visual do sistema de controle postural (vestibular, somatossensorial e motora). Tais ajustes fizeram com que o sistema de controle postural respondesse da mesma forma ao estímulo visual, em função deste sistema ajustar sua freqüência de oscilação à freqüência do estímulo visual, alterando assim a freqüência natural de oscilação deste sistema. De forma geral, os resultados destes estudos indicam que o sistema de controle postural acopla a informação sensorial e, ainda, altera os parâmetros de seu funcionamento às características do estímulo sensorial. Apesar do desvendamento desse processo de funcionamento do sistema de controle postural estar apenas no início, pode-se questionar se essas características de funcionamento são também observadas em idosos e se o relacionamento entre informação sensorial e ação motora ou o acoplamento percepção-ação em idosos é semelhante ao observado em adultos.

\section{RELACIONAMENTO ENTRE INFORMAÇÃO SENSORIAL E AÇÃO MOTORA EM IDOSOS}

Tendo em vista que tanto o sistema motor quanto os sistemas sensoriais vão tendo a capacidade funcional alterada com o envelhecimento, o relacionamento entre informação sensorial e ação motora poderia também ser alterado com o avanço da idade.

Entretanto, poucos são os estudos que diretamente tiveram como objetivo verificar como ocorre este relacionamento em idosos.

Utilizando uma sala móvel, Wade e colaboradores [54] verificaram que idosos respondem com maior oscilação corporal às modificações do fluxo óptico, oscilando mais quando submetidos aos movimentos da sala, quando comparados aos adultos. Segundo estes autores, os idosos oscilam mais quando submetidos aos movimentos do cenário visual devido a vários fatores, onde se destaca a diminuição da capacidade do sistema somatossensorial e vestibular em detectar movimentos corporais. Isto levaria os idosos a necessitar mais das informações visuais para o controle da postura. Por esta razão, suas oscilações corporais seriam maiores quando estes fossem submetidos à manipulação dos estímulos visuais gerados pelo movimento contínuo do cenário visual. Ainda, segundo esses autores, o sistema de controle postural não teria "confiança" em utilizar as infor- mações dos outros sistemas sensoriais em virtude dos déficits apresentados pelos mesmos. Do mesmo modo, Sundermier e colaboradores [49] verificaram que idosos, quando submetidos a movimentos discretos de uma sala móvel, ora para a frente, ora para trás, também apresentavam maior deslocamento do $\mathrm{CP}$ quando comparados aos adultos jovens.

Nestes dois estudos citados acima, a sala móvel foi utilizada apenas como um instrumento que fornecia um estímulo visual com o objetivo de se verificar uma resposta motora. Não houve a preocupação por parte destes autores em entender como ocorre o relacionamento entre informação visual e oscilação corporal. $\mathrm{O}$ interesse em entender como se dá este relacionamento entre percepção e ação em idosos é recente. Ferraz e colaboradores [14] verificaram que o acoplamento entre informação somatossensorial e ações posturais ocorrem de forma diferente em idosos e adultos. Por outro lado, utilizando o paradigma da sala móvel, Polastri e colaboradores [42] não encontraram diferenças na força e na estabilidade do acoplamento entre idosos e adultos. Entretanto, um aspecto interessante neste último estudo foi que, devido à amplitude de deslocamento da sala móvel ter sido grande, os adultos conseguiram discriminar o movimento da sala móvel, enquanto que os idosos não foram capazes de perceber que a sala estava sendo movimentada. Estes autores concluíram que os idosos teriam uma dificuldade maior em discriminar que o estímulo visual era conflitante, por meio de comparação com as outras informações sensoriais. Isto nos permite sugerir que os idosos teriam maior dificuldade em discriminar conflitos sensoriais e esta dificuldade estaria associada a problemas em integrar e comparar as informações sensoriais. Recentemente, Prioli e colaboradores [43] observaram que, durante a manutenção da postura ereta dentro de uma sala móvel movimentada continuamente e com uma amplitude de movimento que não permitiu que os participantes discriminassem qualquer movimento da sala, idosos praticantes e não praticantes de atividade física regular tiveram um acoplamento percepção-ação mais forte que adultos jovens. Estes resultados surpreenderam os autores, pois eles esperavam que o acoplamento entre informação visual e oscilação postural diminuiria com o avanço da idade. Segundo estes autores, a explicação para este fato 
estaria na instabilidade inerente do sistema de controle postural de pessoas idosas. Para eles, os idosos já apresentam alterações no sistema de controle postural que os deixam mais instáveis. Este fato pode ser constatado nos estudos que apontam que os idosos oscilam mais que adultos jovens durante a manutenção da postura ereta $[6,7,21,24,39]$.

Assim, segundo Prioli e colaboradores [43], os idosos, por terem um controle postural menos estável, quando submetidos a um ambiente onde uma modalidade de informação sensorial é mais destacada, não conseguem identificar e resolver o conflito sensorial e, deste modo, se deixam influenciar pela informação sensorial que está sendo manipulada, acoplando a esta informação mais fortemente e dando um peso maior a esta informação. Por outro lado, os adultos jovens conseguem, por meio de uma integração sensorial mais efetiva, minimizar os efeitos provocados pela estimulação sensorial, diminuindo o peso dado à informação manipulada, o que resulta em uma diminuição da influência das oscilações da sala móvel nas ações do sistema de controle postural e, consequentemente, numa diminuição da força do acoplamento entre informação visual e oscilação postural.

Mas o que torna os idosos menos estáveis durante a manutenção da postura ereta? Como citámos anteriormente, alterações estruturais e funcionais ocorrem em todos os canais sensoriais e também no sistema motor, todavia, estas alterações, provavelmente, não são significativas para alterar o comportamento do sistema de controle postural [57]. Se as alterações estruturais e funcionais dos sistemas sensoriais e motor não tornam o sistema de controle postural menos estável, o que provoca tal instabilidade? A causa provável da instabilidade do sistema de controle postural em idosos é a alteração no relacionamento entre informação sensorial e ação motora, representada pela dificuldade que os idosos apresentam em integrar as informações sensoriais, identificar as informações mais relevantes, dando pesos adequados a cada uma das informações de acordo com o contexto, e selecionar a resposta postural mais adequada para manter o corpo equilibrado e na posição desejada [43].

Em suma, os problemas enfrentados por idosos durante o controle postural estariam associados a alterações no relacionamento entre informação sen- sorial e ação motora. Mais especificamente, o problema dos idosos seria integrar as informações sensoriais, utilizando as mais relevantes para determinado contexto, e usar estas informações para, de forma rápida e eficaz, gerar ações motoras necessárias para a manutenção do equilíbrio e orientação posturais. Mas, como as alterações no relacionamento entre informação sensorial e ação motora poderiam explicar o aumento da incidência de quedas em idosos? Antes de tudo devemos observar em quais situações os idosos comumente caem. Segundo Lord e colaboradores [34], a maioria das quedas em idosos ocorre em situações nas quais o equilíbrio postural é desafiado ou perturbado, muitas vezes, em situações em que os idosos estão realizando suas atividades da vida diária. Mais importante, entretanto, é que, quando estas situações ocorrem, os idosos têm que produzir uma resposta motora rápida e precisa, a fim de recuperar o equilíbrio postural. O problema, neste caso, é decidir qual resposta deve ser produzida. O primeiro passo é discriminar corretamente o que está ocorrendo. Isso só é possível por meio do uso das informações sensoriais disponíveis e da integração destas para uma precisa interpretação da situação. Entretanto, os idosos têm dificuldades, conforme apresentado anteriormente, justamente em conseguir uma correta e precisa discriminação da situação de forma rápida. Assim, tendo em vista que os idosos necessitam de um tempo maior para discriminar e responder apropriadamente à situação de perturbação ao equilíbrio, quedas podem ocorrer com uma maior freqüência nesta população. Essa situação pode ser exemplificada em condições nas quais idosos, durante o andar, escorregam ou tropeçam em algum objeto. Nestas situações, os idosos necessitam, primeiramente, detectar este acontecimento, por meio das informações sensoriais disponíveis, integrar tais informações, selecionar a melhor resposta para aquela situação baseada nas informações recebidas e desencadear respostas posturais rápidas e adequadas, para que o equilíbrio corporal seja restabelecido, evitando assim uma queda eminente. Contudo, os idosos têm dificuldade e levam mais tempo para captar, transmitir e integrar as informações sensoriais no sistema de controle postural, têm problemas em utilizar a informação ou as informações mais relevantes para aquele contexto 
específico [15] e necessitam de mais tempo para desencadear as respostas posturais [15, 43]. Com isso, é muito mais difícil para os idosos minimizar os efeitos das perturbações que são impostos ao equilíbrio, e essa seria a principal razão para o aumento da incidência de quedas nesta população. A partir disto, cabe aos estudiosos do movimento humano buscar estratégias de prevenção e de intervenção que visem melhorar este relacionamento entre informação sensorial e ação motora em idosos e, consequentemente, diminuir a incidência de quedas nesta população especifica. Uma das possíveis ações seria a prática regular de atividade física que ameniza a dificuldade de discriminação sensorial e possibilita a produção de respostas posturais apropriadas [43]. Entretanto, muito ainda necessita ser investigado e entendido sobre quais as razões destas alterações com o avanço da idade e quais os efeitos de atividades físicas no funcionamento do controle postural.

\section{CONSIDERAÇÕES FINAIS}

A manutenção do funcionamento efetivo do sistema de controle postural possibilita ao idoso, dentre outras coisas, manter sua independência na realização de atividades motora básicas e das atividades da vida diária. Descobrir o que leva os idosos a diminuir a sua capacidade de controle postural tem sido o objetivo maior de grande parte dos estudiosos desta área. Hoje em dia, o controle postural é visto como sendo o resultado de um relacionamento complexo e dinâmico entre percepção e ação. Neste sentido, alterações no funcionamento do sistema de controle postural em idosos estariam associadas, principalmente, a problemas no relacionamento entre informação sensorial e ação motora. Neste relacionamento estariam inclusas a integração das informações sensoriais, a tomada de decisão sobre qual a melhor estratégia motora a ser utilizada para manter ou buscar a estabilidade e orientação posturais e a utilização de tais informações para a escolha da melhor estratégia motora a ser adotada na busca dos objetivos comportamentais do controle postural. A partir desta proposta, é preciso investigar mais detalhadamente este relacionamento, entendendo todos estes processos, e entendendo o que muda em função do envelhecimento.
Todavia, esta visão de controle postural ainda necessita de muitos outros estudos que busquem entender o relacionamento complexo e dinâmico entre as informações sensoriais e as ações motoras. Não basta entender os processos associados à captação e ao registro dos diferentes tipos de informação sensorial e nem como o sistema muscular gera seus padrões de ativação muscular. Mais importante, a partir deste momento, é entender como as informações sensoriais são utilizadas para a geração de ação motora e como tais ações motoras provocam mudanças no fluxo de informações recebidas pelos seres humanos, compreendendo como este ciclo percepção-ação ocorre durante a realização de movimentos e durante o controle postural.

\section{NOTAS}

${ }^{1}$ Centro de massa de um corpo é o ponto no qual toda a massa do corpo está equilibrada e onde se encontra a resultante de todas as forças que agem sobre o corpo $[25,56]$.

${ }_{2}$ Centro de pressão é o ponto de aplicação da força vertical resultante agindo sobre a superfície de suporte. Representa o resultado das ações do sistema de controle postural e da força da gravidade [12].

\section{CORRESPONDÊNCIA}

José Angelo Barela

Laboratório para Estudos do Movimento

Universidade Estadual Paulista - IB

Departamento de Educação Física

Av. 24 a, 1515

13506-900 Rio Claro, SP

BRASIL

jbarela@rc.unesp.br 


\section{REFERÊNCIAS BIBLIOGRÁFICAS}

1. Barela JA (1997). Development of postural control: the coupling between somatosensory information and body sway. Tese (Doctor of Philosophy) - College Park, University of Maryland, Maryland.

2. Barela JA (2000). Estratégias de controle em movimentos complexos: Ciclo percepção-ação no controle postural. Rev Paul Educ Fís 13: 79-88

3. Barela JA, Jeka JJ, Clark JE (2003). Postural control in children: Coupling to dynamic somatosensory information. Exp Brain Res 150: 434-442

4. Barela JA, Godoi D, Freitas Júnior PB, Polastri PF (2000). Visual information and body sway coupling in infants during sitting acquisition. Inf Behav Dev 23: 285-297

5. Barela JA, Polastri PF, Freitas Júnior PB, Godoi D (2003). Efeito da exposição visual no acoplamento entre informação visual e controle postural em bebês. Rev Paul Educ Fís 17: 16-31

6. Blaszczyk JW, Hansen PD, Lowe DL (1993). Postural sway and perception of the upright stance stability borders. Perception 22: 1333-1341

7. Blaszczyk JW, Lowe DL, Hansen PD (1994). Ranges of postural stability and their changes in the elderly. Gait Posture 2: 11-17

8. Chaimowicz, F (1997). A saúde dos idosos brasileiros às vésperas do século XXI: problemas, projeções e alternativas. Rev Saúde Pública 31: 184-200

9. Collins JJ, De Luca CJ, Burrows A, Lipsitz LA (1995). Agerelated changes in open-loop and closed-loop postural control mechanisms. Exp Brain Res 104: 480-492

10. Dijkstra TMH, Schöner G, Gielen CCAM (1994). Temporal stability of the action-perception cycle for postural control in a moving visual environment. Exp Brain Res 97: 477-486

11. Dijkstra TMH, Schöner G, Giese MA, Gielen CCAM (1994). Frequency dependence of the action-perception cycle for postural control in a moving visual environment: relative phase dynamics. Biol Cybern 71: 489-501

12. Dorfman LJ, Bosley MD (1979). Age-related changes in peripheral and central nerve conduction in man. Neurology 29: 38-44

13. Duarte MD, Zatsiorsky VM (2002). Effects of body lean and visual information on the equilibrium maintenance during stance. Exp Brain Res 146: 60-69

14. Ferraz MA, Barela JA, Pellegrini AM (2001). Acoplamento sensório-motor no controle postural de indivíduos idosos fisicamente ativos e sedentários. Motriz 7: 99-105

15. Freitas Júnior PB (2003). Características comportamentais do controle postural de jovens, adultos e idosos. Dissertação de Mestrado. Universidade Estadual Paulista, Rio Claro, São Paulo, Brasil.

16. Freitas Júnior PB, Barela, JA (2004). Postural Control as a function of self- and object-motion perception. Neurosci Lett 369: 64-68.

17. Gatev P, Thomas S, Kepple T, Hallett M (1999). Feedforward ankle strategy of balance during quiet stance in adults. J. Physiol (Lond.) 514: 915-928

18. Ghez C (1991). Posture. In Kandel ER, Schwartz JH, Jessel TM (ed.) Principles of Neural Science. Norwalk: Appleton \& Longe, $3^{\text {a }}$ ed., 596-608.

19. Gibson JJ (1979). The ecological approach to visual perception. Boston: Houghton Mifflin Company.

20. Gu MJ, Schultz AB, Shepard NT, Alexander NB (1996).
Postural control in young and elderly adults when stance is perturbed: Dynamics. J Biomech 29: 319-329

21. Hageman PA, Leibowitz JM, Blanke D (1995). Age and gender effects on postural control measures. Arch Phys Med Rehabil 76: 961-965

22. Häkkinen K, Pastinen U-M, Karsikas R, Linnamo V (1995). Neuromuscular performance in voluntary bilateral and unilateral contraction and during electrical stimulation in men at different ages. Eur J Appl Physiol 70: 518-527

23. Häkkinen K, Häkkinen A (1991). Muscle cross-sectional area, force production and relaxation characteristics in women at different ages. Eur J Appl Physiol 62: 410-414

24. Hay L, Bard C, Fleury M, Teasdale N (1996). Availability of visual and proprioceptive afferent messages and postural control in elderly adults. Exp Brain Res 108:129-139

25. Horak FB, Macpherson JM (1996). Postural orientation and equilibrium. In Rowell LB, Sherpherd JT (ed.). Handbook of physiology: a critical, comprehensive presentation of physiological knowledge and concepts. New York: Oxford American Physiological Society, 255-292.

26. Horak FB, Shupert CL, Mirka A (1989). Components of postural dyscontrol in elderly: A review. Neurobiol Aging 10: 727-738

27. Jeka JJ, Oie K, Kiemel KS (2000). Multisensory information for human postural control: Integrating touch and vision. Exp Brain Res 134: 107-125

28. Jeka JJ, Oie K, Schöner G, Dijkstra T, Henson E (1998). Position and velocity coupling of postural sway to somatosensory drive. J Neurophysiol 79: 1661-1674

29. Jeka JJ, Schöner G, Dijkstra T, Ribeiro P, Lackner JR (1997). Coupling of fingertip somatosensory information to head and body sway. Exp Brain Res 113: 475-483

30. Jouen F (1988). Visual-proprioceptive control of posture in newborn infants. In Amblard B, Berthoz A, Clarac F (ed.). Posture and gait: development, adaptation and modulation. Paris: Elsevier, 13-22.

31. Lee DN, Aronson E (1974). Visual proprioceptive control of standing in human infants. Percept Psychophys 15: 529 532

32. Lee DN, Lishman JR (1975). Visual proprioceptive control of stance. J Hum Mov Stud 1: 87-95

33. Lishman JR, Lee DN (1973) The autonomy of visual kinaesthesis. Perception 2: 287-294

34. Lord SR, Sherrington C, Menz HB (2001). Falls in older people: Risk factors and strategies for prevention. Cambridge: Cambridge University Press.

35. Maki BE, Holliday PJ, Fernie GR (1990). Aging and postural control: A comparison of spontaneous- and inducedsway balance tests. J Am Geriatr Soc 38: 1-9

36. Maki BE, McIlroy WE (1996). Postural control in the older adult. Clin Geriatr Med 12: 635-658

37. Morasso PG, Schieppati M (1999). Can Muscle Stiffness Alone Stabilize Upright Standing? J. Neurophysiol 83: 1622-1626

38. Nashner LM (1981). Analysis of stance posture in humans. In Towe AL, Luschei ES (ed.). Handbook of Behavioral Neurology vol. 5. New York: Plenum, 527-565.

39. Newell KM, Slobounov SM, Slobounova BS, Molenaar PCM (1997) Short-term non-stationarity and the development of postural control. Gait Posture 6: 56-62

40. Oie KS, Kiemel T, Jeka JJ (2002). Multisensory fusion: 
simultaneous re-weighting of vision and touch for control of human posture. Cog Brain Res 14: 164-176

41. Paulus WM, Straube A, Krafczyk S, Brandt T (1989).

Differential effects of retinal target displacement, changing size, and disparity in control of anterior posterior and lateral body sway. Exp Brain Res 78: 243-252.

42. Polastri PF, Barela AMF, Barela, JA (2001). Controle postural em idosos: relacionamento entre informação visual e oscilação corporal. In IX Congresso Brasileiro de Biomecânica Anais 2: 132-137

43. Prioli AC, Freitas Júnior PB, Barela JA (in press). Physical activity and postural control in elderly: Coupling between visual information and body sway. Gerontology.

44. Rivner MH, Swift TR, Malik K (2001). Influence of age and height on nerve conduction. Muscle Nerve 24: 11341141

45. Schöner G (1991). Dynamic theory of action-perception patterns: the "moving room" paradigm. Biol Cybern 64: 455-462

46. Schöner G, Dijkstra TMH, Jeka JJ (1998). Action-perception patterns emerge from coupling and adaptation. Ecol Psychol 10: 323-346

47. Shumway-Cook A, Woollacott MH (2002). Controle MotorTeoria e Aplicações Práticas. Barueri: Editora Manole.

48. Spirduso WW (1995) Physical dimensions of aging. Champaign: Human Kinetics.

49. Sundermier L, Woollacott MH, Jensen J, Moore S (1996). Postural sensitivity to visual flow in aging adults with and without balance problems. J Gerontol 51: M45-M53

50. Teasdale N, Stelmach GE, Breunig A, Meeuwsen HJ (1991). Age differences in visual sensory integration. Exp Brain Res 85: 691-696

51. Van Asten WNJC, Gielen CCAM, Denier Van Der Gon JJ (1988). Postural adjustments induced by simulated motion of differently structured environments. Exp Brain Res 73: 371-383

52. Vandervoort AA (1992). Effects of ageing on human neuromuscular function: Implications for exercise. Can J Sport Sci 17: 178-184

53. Vandervoort AA, Chesworth BM, Cunninghan DA, Paterson DH, Rechnitzer PA, Koval JJ (1992). Age and Sex Effects on mobility of human ankle. J Gerontol 47: M17M21

54. Wade M, Lindquist R, Taylor J, Treat-Jacobson D (1995). Optical flow, spatial orientation, and the control of posture in the elderly. Psychol Sci 50: 51-58

55. Wann JP, Mon-Williams M, Rushton K (1998). Postural control and co-ordination disorders: The swinging room revisited. Hum Mov Sci 17: 491-513

56. Winter DA (1995). Human balance and posture control during standing and walking. Gait Posture, 3: 193-214

57. Wolfson L, Whipple MA, Derbin CA, Amerman RN, Murphy BS, Tobin JN, Nashner LM (1992). A dynamic posturography study of balance in health elderly. Neurology 42: 2069-2075

58. Woollacott MH, Shumway-Cook A, Nashner LM (1986). Aging and posture control changes in sensory organization and muscular coordination. Int J Aging Hum Dev 23: 97-114

59. Young A, Skelton DA (1994). Applied physiology of strength and power in old age. Int J Sports Med 15: 149151. 